\title{
MARYLAND AND THE DISTRICT OF COLUMBIA.
}

\section{PRIMARY TRAVERSE.}

Washington quadrangle (embracing portions of Laurel, Rockville, East Washington, and West Washington quadrangles).

\section{DISTRICT OF COLUMBIA; MONTGOMERY AND PRINCE GEORGES COUNTIES, MD.}

The following geographic positions were determined by C. B. Kendall in 1903, 1904, 1912, and 1915, and by J. R. Ellis in 1910 and 1912.

The work of 1904, 1910, and 1912, published in Bulletins 245, 276, 496 , and 551, has been readjusted to agree with lines run in 1915 by C. B. Kendall and tied to Soper, Cissel, north and east corners of the District of Columbia, Beal, Maryland Agricultural College, Stabler, and Rockville triangulation stations of the United States Coast and Geodetic Survey.

\section{WASHINGTON QUADRANGLE.}

MARYI,AND.

Along highways near north border of quadrangle.

\begin{tabular}{|c|c|c|c|}
\hline Station. & $\begin{array}{c}0 \\
\text { Latitude. }\end{array}$ & Longitude. & $\begin{array}{l}\text { Distance } \\
\text { between } \\
\text { stations. }\end{array}$ \\
\hline 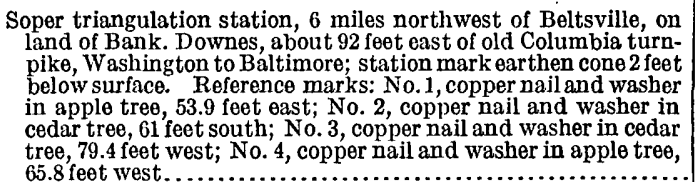 & $\begin{array}{ccc}\circ & \prime & \prime \prime \\
39 & 05 & 11.84\end{array}$ & $\stackrel{\circ}{\circ} \quad \prime \prime \prime$ & Feet. \\
\hline Three corners, 5 feet to corner rail fence, 20 feet south to post..... & 390503.4 & 765704.7 & 911 \\
\hline $\begin{array}{l}\text { Fairland, center of public crossroads at ....................... } \\
\text { Fairland, in west face of south corner of s tone foundation of old } \\
\text { Soper dwelling, in northeast corner of crossroads, } 120 \text { feet north } \\
\text { of center of crossroads; aluininum tablet stamped "Prim. Trav. }\end{array}$ & 390433.6 & 765728 & 3,562 \\
\hline 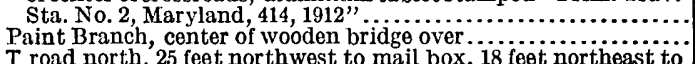 & $\begin{array}{lll}39 & 04 & 34.7 \\
39 & 04 & 38.2\end{array}$ & $\begin{array}{lrr}76 & 57 & 28.9 \\
76 & 58 \cdot 35.4\end{array}$ & 5,261 \\
\hline 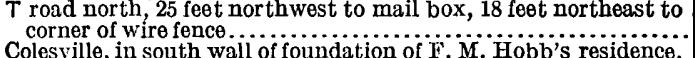 & 390440.6 & 765912.3 & 2,921 \\
\hline 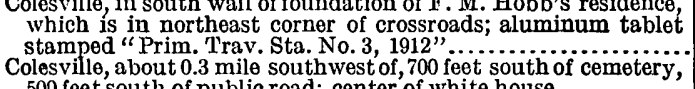 & 390433.2 & 770007.1 & 4,382 \\
\hline $\begin{array}{l}500 \text { feet south of public road; center of white house } \ldots . . . \ldots . . . \\
\text { Colesville, about } 1.1 \text { miles southwest of, } 300 \text { feet south of road; house. }\end{array}$ & $\begin{array}{lll}39 & 04 & 21.7 \\
39 & 03 & 55.4\end{array}$ & $\begin{array}{lll}77 & 00 & 26.3 \\
77 & 01 & 16.8\end{array}$ & $\begin{array}{l}1,913 \\
4,789\end{array}$ \\
\hline $\begin{array}{l}\text { Three corners, } 500 \text { feet south of the northwest mill, } 100 \text { feet south- } \\
\text { west of iron bridge over northwest branch, } 10 \text { feet nor th to Bliss } \\
\text { and Sommers's mail box, } 35 \text { feet southwest to center of plank }\end{array}$ & & & \\
\hline $\begin{array}{l}\text { bridge; public T road south } \\
\text { Second-class road north, at bend of main road to southwest, } 25 \text { feet }\end{array}$ & 390352.2 & 770144.0 & 2,163 \\
\hline $\begin{array}{l}\text { north to gate, } 18 \text { feet west to maple tree........................... } \\
\text { Glenmont, intersection of Union pike and public road northeast } \\
\text { to Colesville, } 40 \text { feet northeast to corner wire fence post, } 35 \text { feet } \\
\text { southeast to telephone pole, } 35 \text { feet southwest to telephone pole }\end{array}$ & 390353.4 & 770223.6 & 3,127 \\
\hline 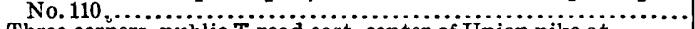 & 390328.8 & & 3,868 \\
\hline $\begin{array}{l}\text { Three corners, public T road east, center of Union pike at......... } \\
\text { Wheaton, on south side of store kept by J. C. Cissel; aluminum }\end{array}$ & 3003106 & 046 & 1,857 \\
\hline $\begin{array}{l}\text { "Prim. Trav. Sta. No. 1, 1915, e } \\
\text { ile southwest of; public road cros }\end{array}$ & & & \\
\hline $\begin{array}{l}\text { Wheaton, } 0.25 \text { mile southwest of; public road crossing ... } \\
\text { Kensington, forks at north edge of, pike north and public }\end{array}$ & 39 & $72+{ }^{2}$ & \\
\hline $\begin{array}{l}\text { northeast, } 20 \text { feet sol } \\
\text { Kensington, intersecti }\end{array}$ & 390200.5 & 770411.1 & 4,632 \\
\hline R. R., 250 feet west of station; center bety & $\begin{array}{llll}39 & 01 & 38.1\end{array}$ & 770422.2 & 2,429 \\
\hline
\end{tabular}


WASHINGTON QUADRANGLE-Continued.

Along Baltimore \& Ohio R. R. from Kensington to Rockville.

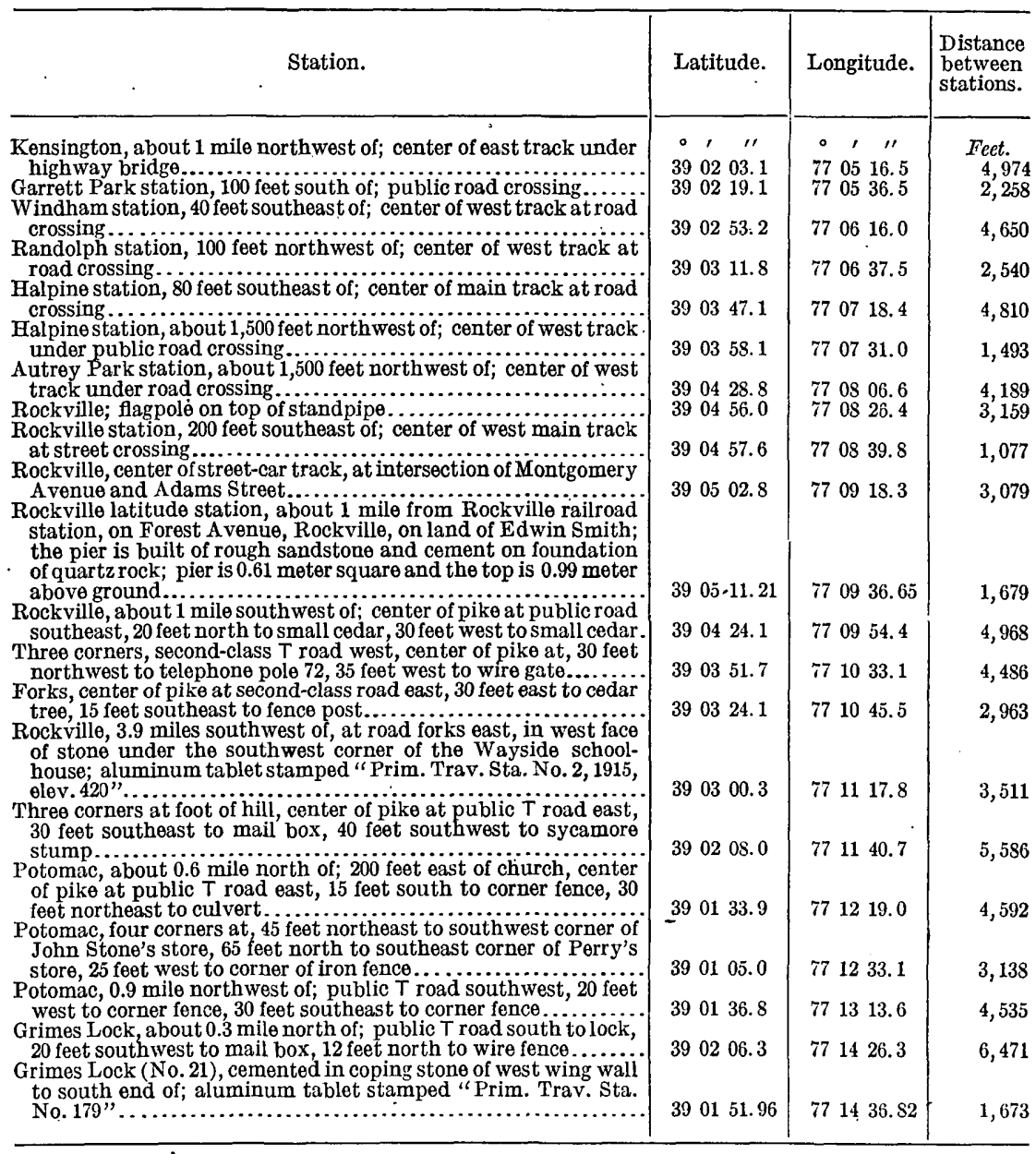

Along highways near north corner of District of Columbia.

Linden, 1,000 feet south of; center of bridge over Baltimore \&

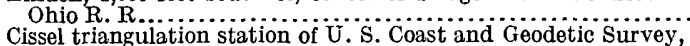
on land of Mr. Cissel on north railroad bank and 600 yards southeast of the house; station mark, an earthenware ale bottle 3 feet underground and granite block 2 feet long and 6 by 6 inches at

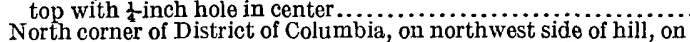
land of Mr. Fenwick. It can be reached by taking Silver Spring road and after passing toll gate by taking left-hand road to $\mathrm{Mr}$. Fenwick's house. Station mark: A 12 by 12 inch stone standing 2 feet out of ground and in good state of preservation......

\begin{tabular}{|c|c|c|}
\hline $\begin{array}{llll}39 & 00 & 19.7\end{array}$ & 770300.3 & \\
\hline $3900 \quad 06.3$ & $\begin{array}{lll}77 & 02 & 39.6\end{array}$ & 2,120 \\
\hline $\begin{array}{llll}38 & 59 & 45.38\end{array}$ & $\begin{array}{lll}7702 & 28.48\end{array}$ & 2,300 \\
\hline
\end{tabular}

Along highways near Maryland Agricultural College.

Lakeland, 50 feet southeast of store, at public road crossing, center

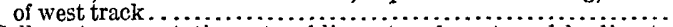
College Avenue station, at public east and west road leading to Maryland Agricultural College, center of (west) track of electric

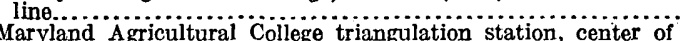
Maryland Agricultural College triangulation station, center of
cupola at south end of old barracks building (destroyed by fire). 
WASHINGTON QUADRANGLE-Continued.

Along highways near Maryland Agricultural College--Continued.

\begin{tabular}{|c|c|c|c|}
\hline Station. & Latitude. & Longitude. & $\begin{array}{l}\text { Distance } \\
\text { between } \\
\text { stations. }\end{array}$ \\
\hline 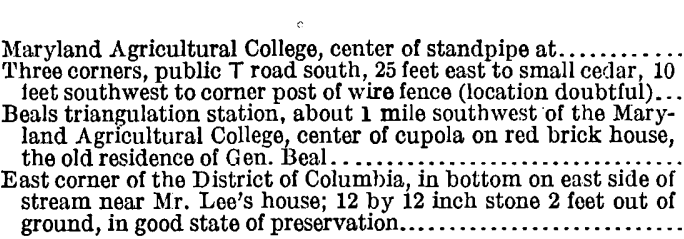 & $\begin{array}{lcc}\circ & \prime \prime \prime \\
38 & 59 & 04.0 \\
38 & 59 & 05.1 \\
38 & 58 & 42.67 \\
38 & 53 & 34.23\end{array}$ & $\begin{array}{ccc}\circ & \prime \prime \prime \\
76 & 56 & 41.3 \\
76 & 56 & 57.1 \\
76 & 57 & 33.84 \\
76 & 54 & 33.94\end{array}$ & $\begin{array}{r}\text { Fect. } \\
411 \\
1,256\end{array}$ \\
\hline
\end{tabular}

Magnetic declination, 1915: Near north border, on line from Soper triangulation station to Kensington, mean declination is $5^{\circ} 03^{\prime} \mathrm{W}$., range $3^{\circ} 55^{\prime} \mathrm{W}$. to $7^{\circ} 02^{\prime} \mathrm{W}$; near north corner of District of Columbia mean declination is $4^{\circ} 54^{\prime} \mathrm{W}$.; near Maryland Agricultural College mean declination is $4^{\circ} 29^{\prime} \mathrm{W}$., range $1^{\circ} 35^{\prime} \mathrm{E}$. to $6^{\circ} 45^{\prime} \mathrm{W}$; along railroad, Kensington to Rockville, mean declination is $7^{\circ} 07^{\prime} \mathrm{W}$.; along highways, from Rockville to Grimes Lock, mean declination is $6^{\circ} 02^{\prime} \mathrm{W}$., range $3^{\circ} 00^{\prime} \mathrm{W}$. to $8^{\circ} 19^{\prime} \mathrm{W}$.

The following 37 geographic positions were determined by J.R. Ellis during the season of 1910 and are based on Coast and Geodetic Survey triangulation stations.

WASHINGTON QUADRANGLE AND VICINITY.

Along the Chesapeake \& Ohio Canal between Georgetown, D. C., and Great Falls, Md.

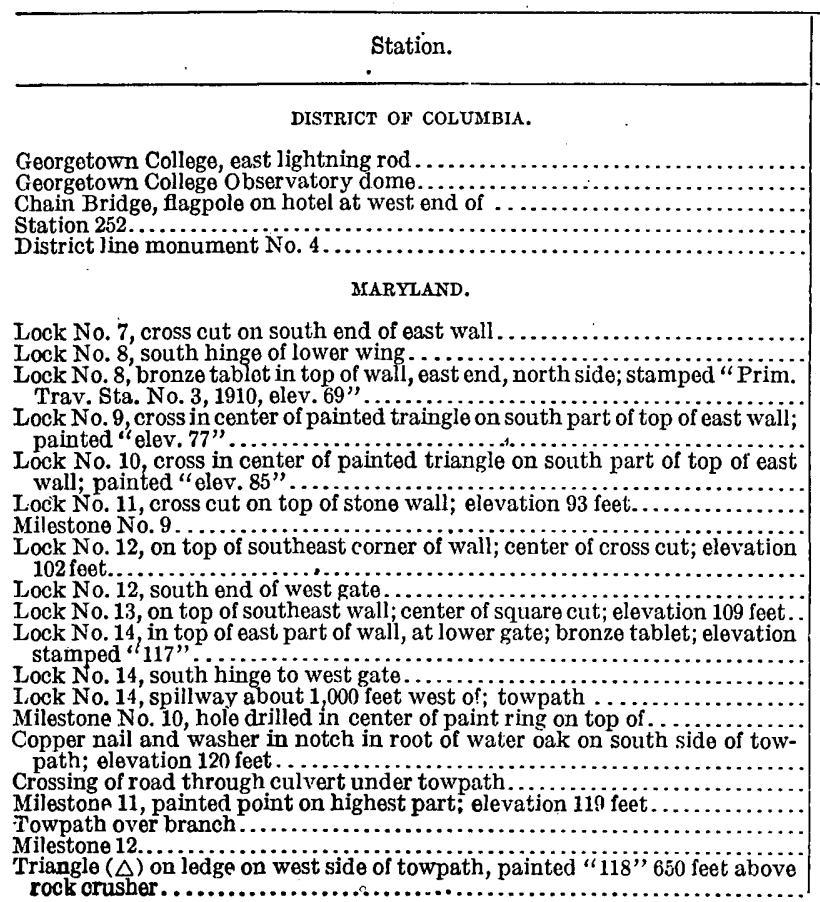

\begin{tabular}{|c|c|}
\hline Latitude. & Longitude. \\
\hline $\begin{array}{lll}\circ & \prime & \prime \prime \\
38 & 54 & 26.13 \\
38 & 54 & 29.94 \\
38 & 55 & 47.95 \\
38 & 55 & 51.01 \\
38 & 56 & 15.28\end{array}$ & $\begin{array}{lll}\circ & \prime & \prime \prime \\
77 & 04 & 22.70 \\
77 & 04 & 39.05 \\
77 & 07 & 02.85 \\
77 & 06 & 46.63 \\
77 & 06 & 57.38\end{array}$ \\
\hline
\end{tabular}

385751.80 $\begin{array}{llll}38 & 58 & 15.00\end{array}$

$\begin{array}{lll}77 & 08 & 20.81\end{array}$ $\begin{array}{llll}38 & 58 \quad 15.27\end{array}$ 385820.04 $\begin{array}{lll}77 & 09 & 38.84\end{array}$ $\begin{array}{llll}38 & 58 & 20.13\end{array}$ $\begin{array}{llll}38 & 58 & 20.31\end{array}$ $\begin{array}{lll}38 & 58 & 20.25\end{array}$ $\begin{array}{llll}38 & 58 & 20.75\end{array}$ $\begin{array}{lll}38 & 58 & 20.66\end{array}$ $\begin{array}{lll}38 & 58 & 20.08\end{array}$ $\begin{array}{llll}38 & 58 & 19.80\end{array}$ $\begin{array}{llll}38 & 58 & 19.39\end{array}$ $\begin{array}{lllll}38 & 58 & 17.82\end{array}$ $\begin{array}{llll}38 & 58 & 15.21\end{array}$ $\begin{array}{llll}38 & 5.5 & 16.79\end{array}$ $\begin{array}{lll}38 & 58 & 23.03\end{array}$ $\begin{array}{llll}38 & 58 & 38.74\end{array}$ $\begin{array}{lll}38 & 58 & 38.38\end{array}$ $\begin{array}{llll}38 & 58 & 42.42\end{array}$ 385857.06 $\begin{array}{llll}77 & 10 & 00.92\end{array}$ $\begin{array}{llll}77 & 10 & 06.74\end{array}$ $\begin{array}{llll}77 & 10 & 18.89\end{array}$ $\begin{array}{llll}77 & 10 & 10.88\end{array}$ 771039.63 $\begin{array}{llll}77 & 10 & 41.20\end{array}$ $7710 \quad 45.20$ $\begin{array}{llll}77 & 10 & 50.85\end{array}$ $\begin{array}{llll}77 & 10 & 52.23\end{array}$ $\begin{array}{llll}77 & 11 & 04.81\end{array}$ $\begin{array}{lll}77 & 11 & 27.38\end{array}$ $\begin{array}{lll}77 & 11 & 44.02\end{array}$ $\begin{array}{llll}77 & 11 & 56.30\end{array}$ 7712 25. 06 $\begin{array}{lll}77 & 13 & 15.72\end{array}$ $\begin{array}{lll}77 & 13 & 26.76\end{array}$ 771352.23 
WASHINGTON QUADRANGLE AND VICINITY-Continued.

Along the Chesapeake \& Ohto Canal between Georgetown, D. C., and Great Falls, Md.-Continued.

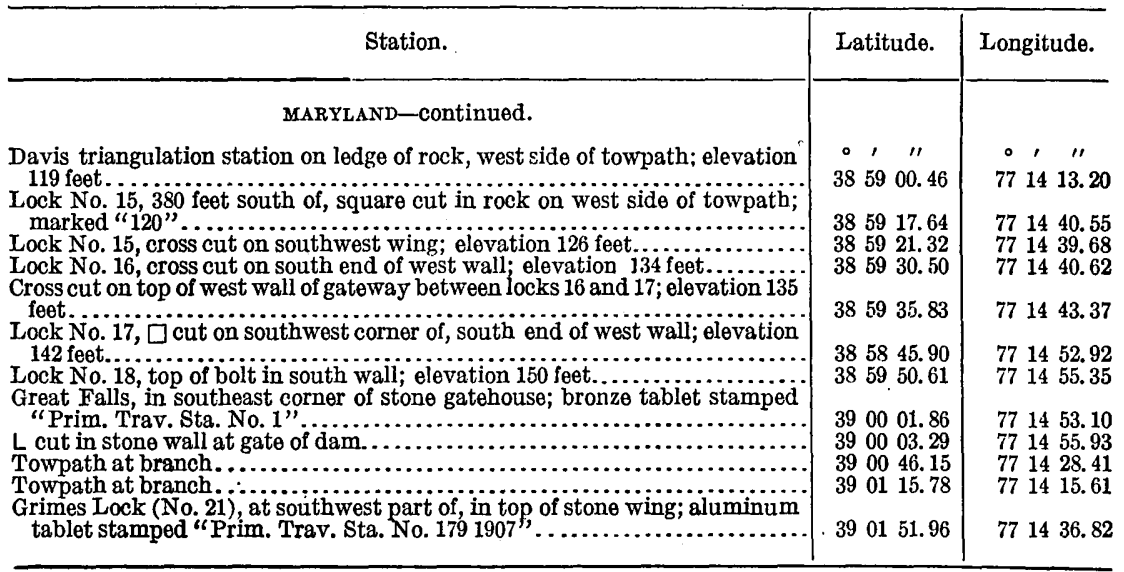

\section{LAUREL QUADRANGLE.}

From Arundel to Bowle.

\begin{tabular}{|c|c|c|}
\hline Station. & Latitude. & Longitude. \\
\hline 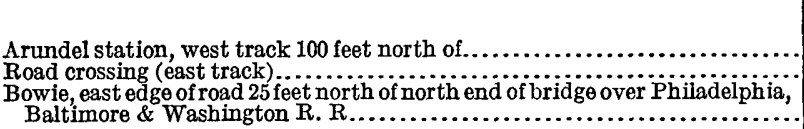 & $\begin{array}{ccc}\circ & \prime & \prime \prime \\
39 & 01 & 57.9 \\
39 & 01 & 03.7 \\
39 & 00 & 29.4\end{array}$ & $\begin{array}{ccc}\circ & \prime & \prime \prime \\
76 & 45 & 12.7 \\
76 & 45 & 54.5 \\
76 & 46 & 44.5\end{array}$ \\
\hline
\end{tabular}

Along highways.

Bowie, three corners near stone residence in northwest part of

White house, three corners, 300 feet east of

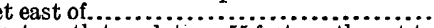
Three corners, telegraph road, 45 feet south to oak tree, 55 feet northwest to wild cherry tree.

Forks of road to west at row of cedars.

Three corners, 22 feet south to fence post, 40 feet north to pear $t$ ree.

Four corners, 40 feet southeast to oak tree, 43 feet southwest to corner of dweil-

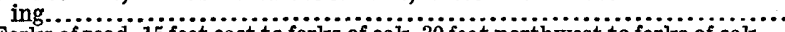
Forks of road, 15 feet east to forks of oak, 30 feet northwest to forks of oak...

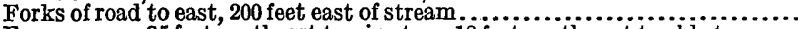
Four corners, 35 feet southeast to pine tree, 18 feet northwest to old stump... Branchville, road crossing.

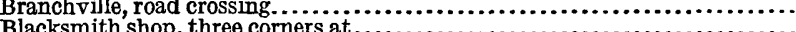

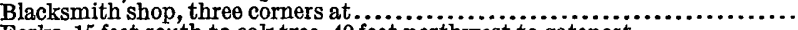
Forks, 15 feet south to oak tree, 40 feet northwest to gatepost.

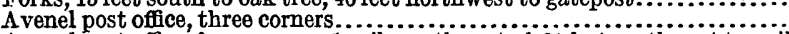

Avenel post office, four corners, 1 mile southwest of, 31 feet northwest to nail

in telephone pole, 30 feet to nail in cedar tree.

Four corners, 30 feet east to north stone gatepost, 23 feet west to corner of Labyrinth's real estate office.

Burnt Mills post office, forks of road 300 feet south Junction of Columbia pike and Bladensburg road at No. 2 tollgate ......... Three corners, 15 feet west to oak tree, 40 feet east to mail box.

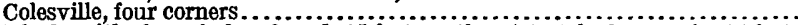

Blacksmith shop, forks of road, 25 feet southeast to telephone pole, 35 feet southwest to telephone pole............................................... Cloverly post office, three corners, 53 feet west to corner of dwelling, 60 feet

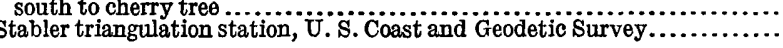

\begin{tabular}{|c|c|}
\hline $\begin{array}{llll}39 & 00 & 38.7 \\
39 & 00 & 34.7\end{array}$ & $\begin{array}{llll}76 & 46 & 55.7 \\
76 & 48 & 05.5\end{array}$ \\
\hline $\begin{array}{llll}39 & 00 & 25.2 \\
39 & 00 & 46.0 \\
39 & 00 & 56.8\end{array}$ & $\begin{array}{llll}76 & 48 & 38.0 \\
76 & 49 & 14.4 \\
76 & 50 & 53.4\end{array}$ \\
\hline $\begin{array}{llll}39 & 01 & 10.6 \\
39 & 00 & 40.7 \\
39 & 00 & 19.9 \\
38 & 59 & 46.5 \\
38 & 59 & 55.5 \\
38 & 59 & 54.1 \\
39 & 00 & 08.6 \\
39 & 00 & 14.7\end{array}$ & $\begin{array}{lll}76 & 51 & 30.1 \\
76 & 52 & 45.2 \\
76 & 53 & 40.4 \\
76 & 54 & 06.3 \\
76 & 55 & 13.4 \\
76 & 55 & 54.4 \\
76 & 57 & 57.4 \\
76 & 58 & 51.9\end{array}$ \\
\hline $\begin{array}{lll}38 & 59 & 58.1\end{array}$ & $76 \quad 59 \quad 44.2$ \\
\hline $\begin{array}{lll}39 & 01 & 12.5 \\
39 & 01 & 50.1 \\
39 & 02 & 25.7 \\
39 & 03 & 18.0 \\
39 & 04 & 32.8\end{array}$ & $\begin{array}{llll}77 & 00 & 46.8 \\
77 & 00 & 19.0 \\
76 & 59 & 28.5 \\
76 & 59 & 44.2 \\
77 & 00 & 07.8\end{array}$ \\
\hline $\begin{array}{lll}39 & 05 & 20.8\end{array}$ & $\begin{array}{llll}77 & 00 & 00.4\end{array}$ \\
\hline $\begin{array}{lll}39 & 06 & 42.6 \\
39 & 07 & 17.7\end{array}$ & $\begin{array}{lll}76 & 59 & 42.8 \\
76 & 59 & 06.5\end{array}$ \\
\hline
\end{tabular}


ROCKVILLE QUADRANGLE.

Along highways.

\begin{tabular}{|c|c|c|}
\hline Station. & Latitude. & Longitude. \\
\hline $\begin{array}{l}\text { Brink post office, three corners, } 1 \text { mile souti of, at Neelsville Presbyterian } \\
\text { Church, } 50 \text { feet enst to wire fence, } 40 \text { feet northwest to vard corner.......... }\end{array}$ & 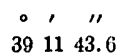 & ○ $\quad{ }^{\prime} \quad \prime \prime \prime$ \\
\hline $\begin{array}{l}\text { Middlebrook, four corners, } 60 \text { feet northwest to store corner, } 45 \text { feet southwest } \\
\text { to stone fence corner }\end{array}$ & & \\
\hline nce corner . .......... & & 8 \\
\hline $\begin{array}{l}\text { Seneca Creek, center of bridge over............ } \\
\text { Three corners, } 40 \text { feet east to tree at fence cor }\end{array}$ & 100 & 7.4 \\
\hline corner............................... & 390927 & 771304.7 \\
\hline $\begin{array}{l}\text { Gaithersburg cemented in north wall at northwest corner of brick store of } \\
\text { Mr. R. A. Young at junction of Frederick Avenue and Chestnut Street, } \\
\text { aluminum toblet stamped "Prim. Trav. Sta. No. 24" }\end{array}$ & & 77120 \\
\hline Gaithersburg, Frederick Avenue crossing, Baltimore \& Ohio R. R., 55 feet & & \\
\hline Thre & $\begin{array}{l}390829.2 \\
390729.6\end{array}$ & $\begin{array}{l}771155.4 \\
771248.9\end{array}$ \\
\hline ers, 30 feet northeast to cedar tree, 35 feet southeast to fence cor & & 771258.9 \\
\hline & 11 & 77132 \\
\hline Three corners, 40 feet north to fence co & & 55.0 \\
\hline $\begin{array}{l}\text { (No. } 2 \text { ), } 3 \text { corners, } 2 \mathrm{~m} \\
36 \text { feet southwest to } \mathrm{n}\end{array}$ & 390206.5 & 771426.3 \\
\hline Grimes tor & & \\
\hline Trav. Sta. No. 179".. & & 36.8 \\
\hline 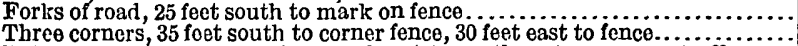 & & 9.5 \\
\hline Pótomac, fou & & \\
\hline feet east to & & 771233.1 \\
\hline $\begin{array}{l}\text { Three corners, } 3 \\
\text { Cabin John Cree }\end{array}$ & & 1.78 \\
\hline outheast to corner smali dwelling, 25 feet south & & \\
\hline theast of, 25 feet east to $\mathrm{w}$ & & 770 \\
\hline 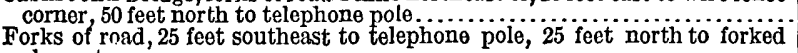 & $3 s$ & 770815.22 \\
\hline Tha, La Reov do & & 7.70 \\
\hline track, 50 feets & & \\
\hline $\begin{array}{l}\text { Georgetown pike, thre } \\
\text { Bethesda street-iar sta }\end{array}$ & 390023.28 & 770551. \\
\hline etown pike, 0.8 mile east & & \\
\hline to telephone pole, 50 feet east to telephone & $\begin{array}{l}3900 \quad 21.57 \\
385946.06\end{array}$ & 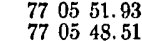 \\
\hline ag, 1 mile south of ............................. & 38 & 77 \\
\hline t alen, 45 feet southwest & & \\
\hline & 390034.55 & 770227.25 \\
\hline 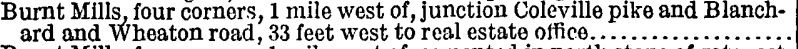 & 390112.5 & 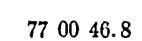 \\
\hline 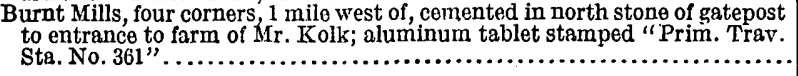 & 390112.6 & 770046.0 \\
\hline
\end{tabular}

WASHINGTON QUADRANGLE.

MARYLAND.

Along City \& S̊uburban. Electric Raliway and highways near east border of quadrangle.

\begin{tabular}{|c|c|c|c|}
\hline Station. & Latitude. & Longitude. & $\begin{array}{l}\text { Distance } \\
\text { between } \\
\text { stations. }\end{array}$ \\
\hline 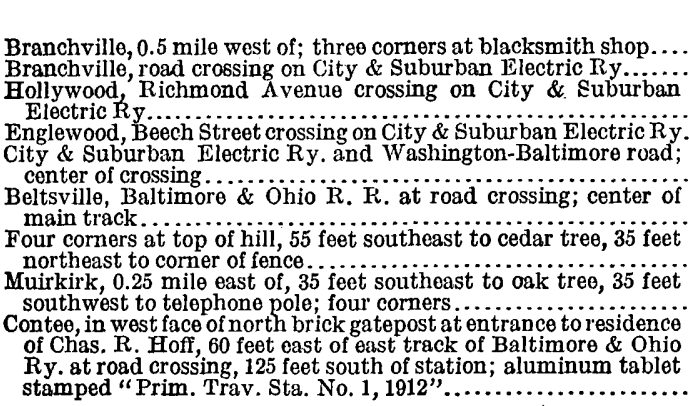 & $\begin{array}{lcc}\circ & \prime \prime \prime \\
38 & 59 & 54.1 \\
38 & 59 & 56.7 \\
39 & 00 & 37.5 \\
39 & 00 & 57.8 \\
39 & 01 & 45.3 \\
39 & 02 & 09.3 \\
39 & 02 & 35.0 \\
39 & 03 & 35.6\end{array}$ & $\begin{array}{cccc}0 & 1 & \prime \prime \\
76 & 55 & 54.4 \\
76 & 55 & 32.3 \\
76 & 55 & 21.3 \\
76 & 55 & 15.8 \\
76 & 54 & 56.7 \\
76 & 54 & 21.7 \\
76 & 53 & 45.0 \\
76 & 52 & 53.4\end{array}$ & $\begin{array}{l}\text { Fect. } \\
\cdots, \ldots, 761 \\
4,220 \\
2,100 \\
5,041 \\
3,682 \\
3,886 \\
7,358\end{array}$ \\
\hline
\end{tabular}


WASHINGTON QUADRANGLE-Continued.

Along hlghways near north border of quadrangle.

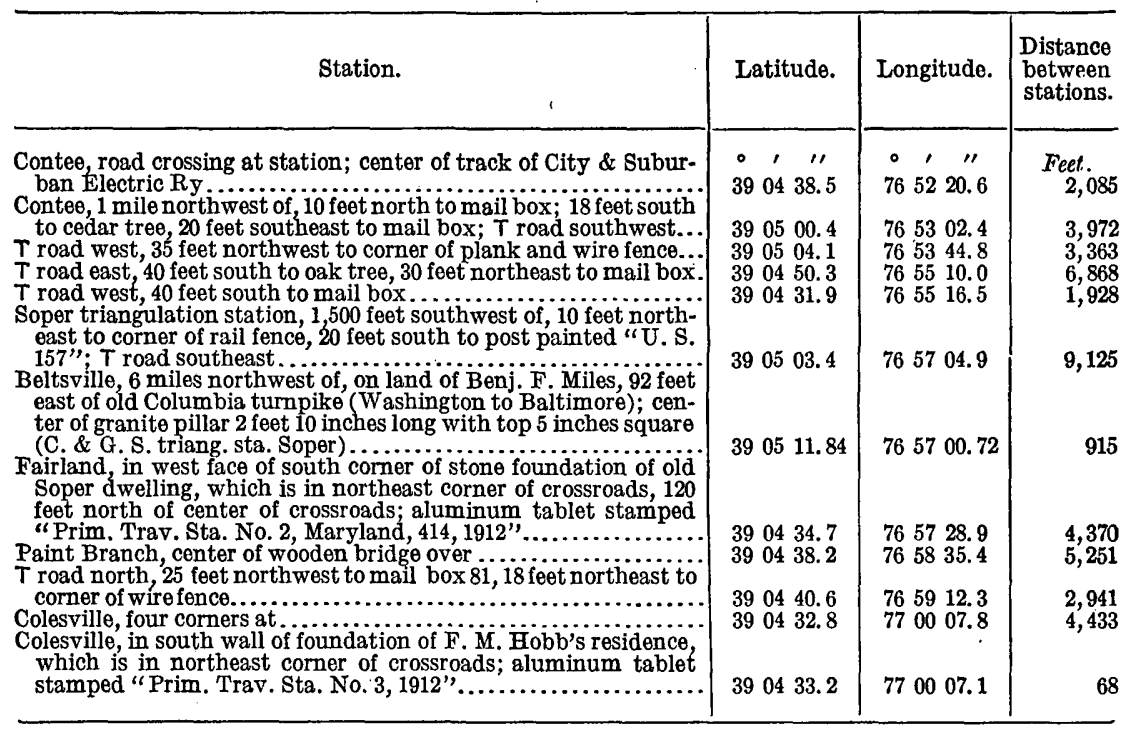

The following geographic positions were established in 1912 by J. R. Ellis. The line starts from an adjusted position 1 mile east of Branchville and follows highways to Lanham, thence along the Pennsylvania Railroad to Ardwick station, thence by highway to Ardmore station, Brightseat, Seat Pleasant, and connects with the east District of Columbia monument. A line starts from an adjusted position 1 mile east of Seat Pleasant, follows public highways through Richie, Forestville, Camp Springs, and Friendly, and connects with the District of Columbia monument at Fox Ferry.

WASHINGTON QUADRANGLE.

MARYLAND.

Along highways and Pennsylvania Railroad near east border of quadrangle.

\begin{tabular}{|c|c|c|c|}
\hline Station. & Latitude. & Longitude. & $\begin{array}{l}\text { Distance } \\
\text { between } \\
\text { stations. }\end{array}$ \\
\hline & 0,11 & $\circ, " 1$ & Feet. \\
\hline 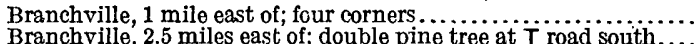 & 385946.5 & 765406.3 & . \\
\hline Branchville, 3 miles east of, at west point of triangle in forks; & 38 & 76 & 7,4 \\
\hline $\begin{array}{l}\text { cross cut in blaze on white-oak tree } \ldots \ldots \ldots \ldots \ldots \ldots \ldots \ldots \ldots \ldots \\
\text { Lanham, } 1.8 \text { miles north of, on north side of field; east point of }\end{array}$ & 385930.5 & 765157.8 & 2,930 \\
\hline triangle at forks.............................................. & 385905.3 & 765145.6 & 2,73 \\
\hline $\begin{array}{l}\text { Lanham, } 1.2 \text { miles north of, at } \log \text { house, } 22 \text { feet west to blaze on } \\
\text { small pine tree; center of crossroads...1. } \\
\text { I anham, } 0.3 \text { mile northeast of, in south end of retaining wail of }\end{array}$ & 385840.5 & $7651 \quad 19.3$ & 3,253 \\
\hline $\begin{array}{l}\text { west end of bridge over Pennsylvania } \mathrm{R}, \mathrm{R} . ; \text { aluminum tablet } \\
\text { stamped "Prim. Trav. Sta. No. 1, 1912" }\end{array}$ & $\begin{array}{lll}38 & 57 & 50.7\end{array}$ & & 0 \\
\hline $\begin{array}{l}\text { Lanham, east track of Pennsylvania } R \text {. } R \text {. at road crossing.... } \\
\text { Ardwick station, middle track of Pennsylvania } R \text {. } R \text {, at road }\end{array}$ & $\begin{array}{lll}38 & 57 & 34.0\end{array}$ & 76 & 01 \\
\hline 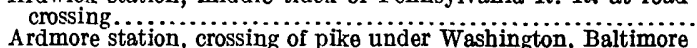 & 38564 & 765235.4 & 6,57 \\
\hline \& Annapolis R. R. (ele & 385619.0 & 765126.6 & 5,85 \\
\hline
\end{tabular}


WASHINGTON QUADRANGLE-Continued.

Along highways and Pennsylvania Rallroad near east border of quadrangle-Continued.

\begin{tabular}{|c|c|c|c|}
\hline Station. & Latitude. & Iongitude. & $\begin{array}{l}\text { Distance } \\
\text { between } \\
\text { stations. }\end{array}$ \\
\hline 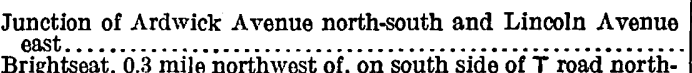 & $\begin{array}{cc}\circ \quad \prime \prime \\
385548.5\end{array}$ & $\begin{array}{cc}\circ \quad \prime \prime \\
76 & 5135.4\end{array}$ & $\begin{aligned} & \text { Feet. } \\
- & 3,162\end{aligned}$ \\
\hline 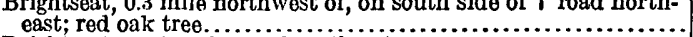 & 385507.4 & 765141.4 & 4,189 \\
\hline $\begin{array}{l}\text { Brightseat, center of } \mathrm{T} \text { road northeast } \\
\text { Brightseat, } 1 \text { mile south of; cross on top of stone monument in }\end{array}$ & 385500.3 & 765120.2 & 1,820 \\
\hline Brightseat, 1.5 miles east of; center of intersection of central & 385425.7 & 765111.2 & 3,569 \\
\hline 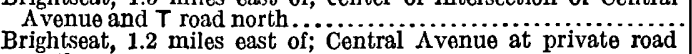 & 385328.5 & 765102.5 & 5,825 \\
\hline 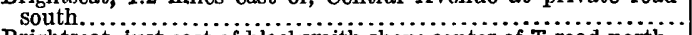 & 385325.2 & 765124.0 & 1,737 \\
\hline Brightseat, just east of blacksmith shop; center of $T$ road north.. & $\begin{array}{llll}38 & 53 & 18.6 \\
38 & 53 & 12 & 5\end{array}$ & 765240.6 & 6,091 \\
\hline $\begin{array}{l}\text { District station, } 0.8 \text { mile southeast of; center of crossroads at store.. } \\
\text { East corner monument of District of Columbia.................. }\end{array}$ & $\begin{array}{lll}38 & 53 & 12.5 \\
38 & 53 & 34.23\end{array}$ & $\begin{array}{lll}76 & 53 & 54.3 \\
76 & 54 & 33.94\end{array}$ & $\begin{array}{l}5,860 \\
3,828\end{array}$ \\
\hline $\begin{array}{l}\text { Richie station, } 0.8 \text { mile northwest of; road crossing under chesa } \\
\text { peake Beach } R . \mathbf{R} . \ldots \ldots \ldots \ldots \ldots \ldots \ldots \ldots \ldots \ldots \ldots \ldots \ldots \ldots \ldots \ldots\end{array}$ & 385242.7 & $7651 \quad 46.1$ & \\
\hline Richie station, just west of; road crossing on Chesapeake Beach & & & \\
\hline 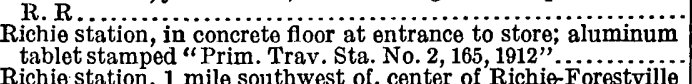 & $\begin{array}{l}385211.2 \\
385207.5\end{array}$ & $\begin{array}{ll}76 & 5121.1 \\
76 & 5120.3\end{array}$ & $\begin{array}{r}3,764 \\
372\end{array}$ \\
\hline $\begin{array}{l}\text { pike at } T \text { road east. . . } \\
\text { Forestville, } 20 \text { feet west to "Ele. } 280.1 \% \text { on telephone pole, } 36 \text { feet } \\
\text { north to corner post; crossing of Marlboro turnpike and north- }\end{array}$ & 385136.9 & 765218.0 & 5,511 \\
\hline 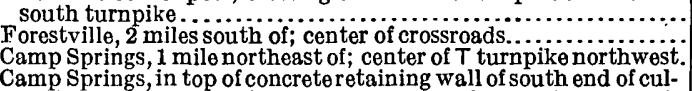 & $\begin{array}{lll}38 & 50 & 40.3 \\
38 & 49 & 03.0 \\
38 & 48 & 41.5\end{array}$ & $\begin{array}{lll}76 & 52 & 27.2 \\
76 & 52 & 40.4 \\
76 & 53 & 44.2\end{array}$ & $\begin{array}{l}5,776 \\
9,905 \\
5,491\end{array}$ \\
\hline 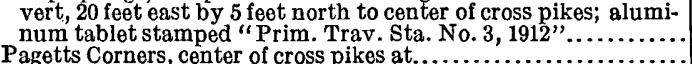 & 384813.5 & 765425.9 & 4,357 \\
\hline 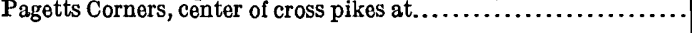 & 384735.2 & 765544.3 & 7,314 \\
\hline
\end{tabular}

Along highway near south border of quadrangle.

Schoolhouse, center of crossroads

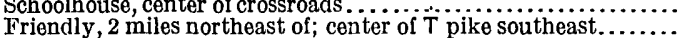

Friendly, 1.25 miles northeast of, 20 feet south by 15 feet west to

Steed's mail box; center of Camp Springs-Friendly road at road east.

Hyland Harmon's mail box on south side of forks . . . . . . . . . . . . . . . .

Friendly, 20 feet south to Geo. M. Boland's mail box; center of $T$

road northeast.

Friendly, 1 mile west by 0.5 mile north of; center of $\mathrm{T}$ turnpike

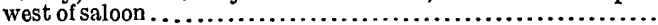
New Glatz, 1 mile south of; forks of road

New Glatz, 20 feet west by 10 feet south to corner of yard fence;

$T$ road west at store.

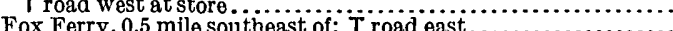

Fox Ferry, District of Columbia monument

\begin{tabular}{llll|llll|l}
38 & 47 & 15.1 & 76 & 56 & 19.3 & 3,424 \\
38 & 46 & 35.2 & 76 & 57 & 07.9 & 5,583 \\
& & & & & \\
38 & 45 & 42.3 & 76 & 57 & 23.6 & \\
38 & 45 & 20.4 & 76 & 57 & 53.3 & 5,497 \\
& & & & & & \\
38 & 45 & 06.0 & 76 & 58 & 42.9 & \\
& & & & & \\
38 & 45 & 30.5 & 76 & 59 & 46.2 & \\
38 & 45 & 40.3 & 77 & 00 & 18.7 & 5,590 \\
& & & & & & \\
38 & 46 & 38.2 & 77 & 00 & 22.4 & \\
38 & 47 & 48.1 & 77 & 01 & 10.9 & 5,866 \\
38 & 48 & 05.57 & 77 & 01 & 35.39 & $\mathbf{8}, 039$ \\
& & & & &
\end{tabular}


